\title{
Diffusion capacity abnormalities for carbon monoxide in patients with COVID-19 at 3-month follow-up
}

\author{
Wei Qin ${ }^{1,11}$, Shi Chen ${ }^{1,11}$, Yunxia Zhang ${ }^{2,3,4,11}$, Fen Dong ${ }^{5,11}$, Zhu Zhang ${ }^{2,3,4,11}$, Bingzhu Hu ${ }^{1}$, Ziyang Zhu ${ }^{1}$, \\ Fajiu $\mathrm{Li}^{1}$, Xiaojiang Wang ${ }^{1}$, Yimin Wang ${ }^{2,3,4}$, Kaiyuan Zhen ${ }^{2,3,4}$, Jing Wang ${ }^{6}$, YuLei Wan ${ }^{7}$, Hongbo $\mathrm{Li}^{7}$, \\ Ismaïl Elalamy ${ }^{8,9}$, Chenghong $\mathrm{Li}^{1,12}$, Zhenguo Zhai ${ }^{2,3,4,12}$ and Chen Wang ${ }^{2,3,4,10}$
}

${ }^{1}$ Dept of Pulmonary and Critical Care Medicine, Affiliated Hospital of Jianghan University, Wuhan, China. ${ }^{2}$ Dept of Pulmonary and Critical Care Medicine, Center of Respiratory Medicine, China-Japan Friendship Hospital, Beijing, China. ${ }^{3}$ Institute of Respiratory Medicine, Chinese Academy of Medical Sciences, Beijing, China. ${ }^{4}$ National Clinical Research Center for Respiratory Diseases, Beijing, China. ${ }^{5}$ Institute of Clinical Medical Sciences, China-Japan Friendship Hospital, Beijing, China. ${ }^{6}$ Institute of Basic Research, Chinese Academy of Medical Sciences, Beijing, China. ${ }^{7}$ Dept of Radiology, Affiliated Hospital of Jianghan University, Wuhan, China. ${ }^{8}$ Hematology and Thrombosis Center, Tenon University Hospital, INSERM UMRS 938, Sorbonne University, Paris, France. ${ }^{9}$ The First I.M. Sechenov Moscow State Medical University, Moscow, Russia. ${ }^{10}$ Peking Union Medical College, Chinese, Academy of Medical Sciences, Beijing, China. ${ }^{11}$ These authors contributed equally as co-first authors. ${ }^{12}$ These authors contributed equally to this article as lead authors and supervised the work.

Corresponding author: Zhenguo Zhai (zhaizhenguo2011@126.com)

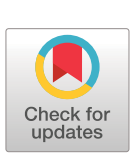

Copyright $\odot$ ERS 2021

This version is distributed under the terms of the Creative Commons Attribution Non-Commercial Licence 4.0 .

This article has supplementary material available from erj. ersjournals.com

Received: 29 Sept 2020 Accepted: 15 Dec 2020

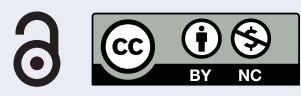

Shareable abstract (@ERSpublications)

COVID-19 patients present with impaired $D_{\text {LCo }}$ at 90 days after discharge, particularly severe patients. Chest CT TSS $>10.5$ and ARDS occurrence are associated with impaired $D_{\text {LCO. Pulmonary }}$ interstitial damage may contribute to the impaired $D_{\mathrm{Lco}}$. https://bit.ly/2JevUtm

Cite this article as: Qin W, Chen S, Zhang Y, et al. Diffusion capacity abnormalities for carbon monoxide in patients with COVID-19 at 3-month follow-up. Eur Respir J 2021; 58: 2003677 [DOI: 10.1183/ 13993003.03677-2020].

\section{Abstract}

Objective To evaluate pulmonary function and clinical symptoms in coronavirus disease 2019 (COVID19) survivors within 3 months after hospital discharge, and to identify risk factors associated with impaired lung function.

Methods and material COVID-19 patients were prospectively followed-up with pulmonary function tests and clinical characteristics for 3 months following discharge from a hospital in Wuhan, China between January and February 2020.

Results 647 patients were included. 87 (13\%) patients presented with weakness, 63 (10\%) with palpitations and 56 (9\%) with dyspnoea. The prevalence of each of the three symptoms were markedly higher in severe patients than nonsevere patients ( $19 \%$ versus $10 \%$ for weakness, $\mathrm{p}=0.003$; $14 \%$ versus $7 \%$ for palpitations, $\mathrm{p}=0.007 ; 12 \%$ versus $7 \%$ for dyspnoea, $\mathrm{p}=0.014$ ). Results of multivariable regression showed increased odds of ongoing symptoms among severe patients (OR 1.7, 95\% CI 1.1-2.6; $\mathrm{p}=0.026$ ) or patients with longer hospital stays (OR 1.03, 95\% CI 1.00-1.05; p=0.041). Pulmonary function test results were available for 81 patients, including 41 nonsevere and 40 severe patients. In this subgroup, 44 (54\%) patients manifested abnormal diffusing capacity of the lung for carbon monoxide $\left(D_{\mathrm{LCO}}\right)$ (68\% severe versus $42 \%$ nonsevere patients, $\mathrm{p}=0.019$ ). Chest computed tomography $(\mathrm{CT})$ total severity score $>10.5$ (OR 10.4, 95\% CI 2.5-44.1; $\mathrm{p}=0.001$ ) on admission and acute respiratory distress syndrome (ARDS) (OR 4.6, 95\% CI 1.4-15.5; $\mathrm{p}=0.014$ ) were significantly associated with impaired $D_{\text {LCO }}$. Pulmonary interstitial damage may be associated with abnormal $D_{\text {LCO. }}$.

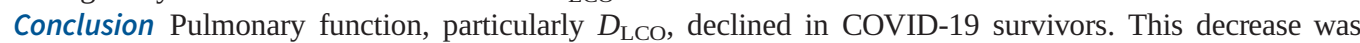
associated with total severity score of chest CT $>10.5$ and ARDS occurrence. Pulmonary interstitial damage might contribute to the imparied $D_{\text {LCO }}$.

\section{Introduction}

The epidemic of coronavirus disease 2019 (COVID-19) has had devastating effects. Patients surviving hospitalisation are frequently reported to have pulmonary sequelae. It is challenging to evaluate lung function 
throughout COVID-19 progression because of the difficulty related to infection control risks in obtaining lung function testing during this contagious pandemic disease [1].

There are short reports of lung function of COVID-19 patients at discharge and at 30 days post-discharge $[2,3]$. Reduced lung function was demonstrated in survivors of severe acute respiratory syndrome (SARS) and Middle East respiratory syndrome (MERS) up to 6 months following hospital discharge [4, 5]. SARS patients were reported to have a mild decrease in diffusing capacity of the lung for carbon monoxide ( $D_{\text {LCO }}$ ) 6-8 weeks after discharge, with improving lung function over time [6]. We performed a prospective cohort study to identify main sequelae and lung function changes in hospitalised severe acute respiratory syndrome coronavirus 2 (SARS-CoV-2) patients during 3-month follow-up.

\section{Methods}

\section{Study design}

This was a prospective cohort study performed at the Affiliated Hospital of Jianghan University (Wuhan, China). COVID-19 was confirmed and diagnosed according to Chinese management guideline for COVID-19 [7]. Nucleic acid tests were provided for SARS-CoV-2 by real-time reverse-transcriptase PCR assays. Severity was defined by the World Health Organization guideline for COVID-19 [8]. Severe pneumonia refers to fever or suspected respiratory infection, plus one of respiratory rate $>30$ breaths $\cdot \mathrm{min}^{-1}$, severe respiratory distress or peripheral oxygen saturation $\leqslant 93 \%$ on room air. A total of 749 COVID-19 patients with full data available were admitted from January to February 2020. Of those, 81 patients died within 3 months and 21 patients were lost during follow-up. 647 patients were followed-up for 3 months after discharge. Patients' clinical baseline details, major clinical characteristics and lung function within 3 months' follow-up were recorded. Ethics approval was obtained from the medical ethics committee of Jianghan University Affiliated Hospital and China-Japan Friendship Hospital (WHSHIRB-K-2020015). Before data collection, we obtained patients' consent.

\section{Pulmonary function tests}

81 (13\%) patients underwent pulmonary function testing (PFT) at 3 months after discharge. PFT was performed by a professional doctor with 20 years of experience using the MasterScreen PFT system (Jaeger, Hoechberg, Germany) at the 3-month follow-up visit. The recorded parameters are as follows: total lung volume (TLC), residual volume, forced vital capacity (FVC), forced expiratory volume in $1 \mathrm{~s}\left(\mathrm{FEV}_{1}\right)$, $\mathrm{FEV}_{1} / \mathrm{FVC}$ ratio, maximum mid-expiratory flow (MMEF) and $D_{\mathrm{LCO}}$.

\section{Chest computed tomography visual quantitative evaluation}

Chest computed tomography (CT) scans were performed on 16- or 64-multidetector CT scanners (GE LightSpeed 16; GE Healthcare, Wauwatosa, WI, USA or Somatom Sensation 64; Siemens Healthcare, Malvern, PA, USA). All patients underwent chest CT scan at admission. In addition, 45 out of 81 patients who had PFT had chest CT scan at 3 months after discharge. Two experienced radiologists (YL Wan and $\mathrm{HB} \mathrm{Li}$ ) reviewed CT images without knowledge of mild or severe, normal or reduced $D_{\text {LCO }}$. Pulmonary interstitial changes on follow-up chest CT graph was defined as a combination of findings including fibrous stripe, ground-glass opacity, consolidation, subpleural curvilinear shadow, coarse reticular pattern and traction bronchiectasis [9]. Meanwhile, main pulmonary artery (MPA), ascending aorta (AAo) diameters and the MPA/AAo ratio were measured to evaluate the relationship between pulmonary vascular disease and impaired $D_{\text {LCO }}[10]$. To explore the relationship between impaired $D_{\text {LCO }}$ and radiographic changes, we conducted chest CT total severity score (TSS), which was evaluated by percentage of involvement in each lobe and overall lung. The percentage of the lobar involvement in each of the five lung lobes were classified in five levels: 0 (0\%), 1 (1-25\%), 2 (26-50\%), $3(51-75 \%)$ and 4 (76-100\%). The TSS was obtained by adding the five lobar scores [11].

\section{Statistical analysis}

Data were expressed as number (\%) for categorical variables, mean \pm sD when they were normally distributed and median (interquartile range (IQR)) when they had skewed distributions. t-test, Mann-Whitney U-test, Chi-squared test or Fisher's exact test were used to compare differences in characteristics and pulmonary function between groups of different characteristics. We used univariable and multivariable logistic regression models to explore the risk factors associated with the occurrence of sequelae and impaired lung function. To avoid overfitting in the multivariable logistic regression model, we chose just four variables for analysis considering the total number of impaired $D_{\text {LCO }}(n=41)$ in our study. The receiver operating characteristic (ROC) curve and the area under the curve were used to assess the predicted value of TSS for impaired $D_{\text {LCO }}$. All statistical analyses were performed using SPSS (version 24; IBM, Armonk, NY, USA) and Prism (version 8.0.1; GraphPad, San Diego, CA, USA) with two-tailed $\mathrm{p}<0.05$. 
Results

\section{Patient characteristics}

647 COVID-19 patients attended the 3-month follow-up visit after hospital discharge, including 399 nonsevere patients and 248 severe patients (supplementary figure S1). The mean \pm SD age was $58 \pm 15$ years, with $44 \%$ being male. The sequential organ failure assessment (SOFA) score on admission was 1 (IQR $0-2$ ). The top three comorbidities were hypertension (30\%), diabetes (11\%) and chronic respiratory disease (6\%). Moreover, severe patients were significantly older and had higher SOFA score at admission (supplementary table S1).

\section{The residual symptoms at 3-month follow-up}

Ongoing symptoms for COVID-19 at the 3-month follow-up visit were weakness, palpitations, dyspnoea, cough, lower limb oedema, chest pain and haemoptysis. 87 (13\%) patients presented with fatigue in their daily lives, 63 (10\%) with palpitations and 56 (9\%) with dyspnoea. Prevalences of the three symptoms in patients with severe COVID-19 were markedly higher than those in the nonsevere patients (19\% versus $10 \%$ for weakness, $\mathrm{p}=0.003 ; 14 \%$ versus $7 \%$ for palpitation, $\mathrm{p}=0.007 ; 12 \%$ versus $7 \%$ for dyspnoea, $\mathrm{p}=0.014$ ) (supplementary table S1). In supplementary table S2, we compare the clinical characteristics between COVID-19 patients who had sequelae or not. The main differences between these two groups were disease severity, inpatient days, inflammation and coagulant disorder on admission. In univariable analysis, odds of sequelae were significantly higher in severe patients with long hospital stay. In addition, higher white blood cell count, higher hypersensitive C-reactive protein and abnormal D-dimer levels were associated with the occurrence of sequelae. In the multivariable model, severity and inpatient days were significantly associated with the occurrence of sequelae (supplementary table S3).

\section{Lung function at 3-month follow-up}

81 COVID-19 patients were assessed for pulmonary function test at 3 months after discharge, including 41 nonsevere and 40 severe patients. In this subgroup of the cohort, the mean \pm sD age was $59 \pm 14$ years, 34 (42\%) patients were male and the mean \pm sD body mass index was $23.87 \pm 3.18 \mathrm{~kg} \cdot \mathrm{m}^{-2}$. As shown in table 1, pulmonary function was impaired in 61 (75\%) out of 81 survivors. Eight (10\%) patients had reduced TLC; 17 (21\%) patients had decreased FVC; in five (6\%) patients, $\mathrm{FEV}_{1} / \mathrm{FVC}$ ratio was < $70 \%$; and in 44 (54\%) patients, $D_{\text {LCO }}$ was $<80 \%$ predicted. To determine whether abnormal lung function was associated with disease severity, we compared characteristics and pulmonary function parameters between nonsevere and severe cases. There were no significant differences in TLC, FVC, FEV $1, \mathrm{FEV}_{1} / \mathrm{FVC}, \mathrm{MMEF}$ at 25$75 \%$ of $\mathrm{FVC}$ or $D_{\mathrm{LCO}}$ to alveolar volume ratio, according to the spirometry between nonsevere and severe patients. However, significant difference was found for $D_{\mathrm{LCO}}$, which was $<80 \%$ pred for $68 \%$ of severe patients compared to $42 \%$ of nonsevere patients $(\mathrm{p}<0.05)$ (figure 1 ).

44 out of 81 COVID-19 patients had impaired $D_{\text {LCO }}$. To figure out the differences between normal and impaired $D_{\text {LCO }}$ patients, we compared clinical characteristics between two groups in table 2 . We found that parameters including severity, chest CT TSS, lymphocyte count, MPA diameter on admission and acute respiratory distress syndrome (ARDS) were higher in patients with impaired $D_{\mathrm{LCO}}$, and the difference between two groups was statistically significant.

TSS of chest CT, pulmonary interstitial damage, vascular disease and impaired $D_{\text {Lco }}$

All patients underwent chest CT scan at admission; in 90\% of patients, lesions could be seen in the bilateral lung on admission and there was no statistical difference between the normal and impaired $D_{\mathrm{LCO}}$ groups $(\mathrm{p}=0.459)$. To evaluate the effect of CT assessment on $D_{\mathrm{LCO}}$ decline in patients with COVID-19, we calculated CT TSS for all patients. The median (IQR) TSS was 9 (5-13) at admission. We created a ROC curve to explore cut-off of TSS to predict abnormal $D_{\mathrm{LCO}}$. We found that the cut-off of TSS was 10.5 on admission; the area under the curve was 0.765 (95\% CI $0.663-0.867 ; \mathrm{p}<0.001)$, with sensitivity 64\% (95\% CI 49-76\%) and specificity 84\% (95\% CI 69-92\%) (figure 2).

45 out of 81 patients who underwent PFT had a chest CT scan at 3-month follow-up after discharge. To determine whether pulmonary interstitial damage contributed to impaired $D_{\mathrm{LCO}}$ or not, we analysed pulmonary CT changes at 3 months after discharge. It was found that patients with abnormal $D_{\text {LCO }}$ were more likely to have interstitial damage, especially manifesting the signs of traction bronchiectasis, subpleural curvilinear shadow and coarse reticular pattern, indicating that pulmonary interstitial damage may contribute to impaired $D_{\text {LCO }}$ in COVID-19 patients (table 3). When exploring the relationship between vascular disease and impaired $D_{\mathrm{LCO}}$, no significant differences were found at 3 months after discharge. 
TABLE 1 Lung function in severe and nonsevere coronavirus disease 2019 patients

\begin{tabular}{|c|c|c|c|c|}
\hline & All & Nonsevere & Severe & p-value \\
\hline Patients & 81 & 41 & 40 & \\
\hline Age years & $59 \pm 14$ & $55 \pm 15$ & $63 \pm 12$ & 0.022 \\
\hline Male & $34(42)$ & $17(42)$ & $17(43)$ & 0.925 \\
\hline $\mathrm{BMI} \mathrm{kg} \cdot \mathrm{m}^{-2}$ & $23.87 \pm 3.18$ & $23.72 \pm 3.41$ & $24.04 \pm 2.95$ & 0.677 \\
\hline \multicolumn{5}{|l|}{ Comorbidities } \\
\hline Hypertension & $23(28)$ & $12(29)$ & $11(28)$ & 0.860 \\
\hline $\mathrm{CHD}$ & $5(6)$ & $2(5)$ & $3(8)$ & 0.675 \\
\hline Diabetes & $7(9)$ & $2(5)$ & $5(13)$ & 0.264 \\
\hline CRD & $6(7)$ & $1(2)$ & $5(13)$ & 0.201 \\
\hline Tumour & $2(3)$ & $2(5)$ & $0(0)$ & 0.494 \\
\hline Arrhythmia & $2(3)$ & $2(5)$ & $0(0)$ & 0.494 \\
\hline \multicolumn{5}{|l|}{ Lung function } \\
\hline TLC \% pred & $99.25 \pm 24.44$ & $102.15 \pm 26.25$ & $96.28 \pm 22.38$ & 0.288 \\
\hline TLC $<80 \%$ pred & $8(10)$ & $1(2)$ & $7(18)$ & 0.029 \\
\hline RV \% pred & $144.85 \pm 66.75$ & $154.78 \pm 76.75$ & $134.68 \pm 53.73$ & 0.176 \\
\hline FVC $\%$ pred & $89.73 \pm 13.25$ & $90.79 \pm 14.75$ & $88.65 \pm 11.59$ & 0.469 \\
\hline FVC $<80 \%$ pred & $17(21)$ & $9(22)$ & $8(20)$ & 0.829 \\
\hline $\mathrm{FEV}_{1} \%$ pred & $93.95 \pm 11.32$ & $94.66 \pm 11.33$ & $93.23 \pm 11.41$ & 0.573 \\
\hline $\mathrm{FEV}_{1}<80 \%$ pred & $5(6)$ & $2(5)$ & $3(8)$ & 0.675 \\
\hline $\mathrm{FEV}_{1} / \mathrm{FVC}$ & $87.23 \pm 10.06$ & $87.63 \pm 9.62$ & $86.82 \pm 10.59$ & 0.719 \\
\hline $\mathrm{FEV}_{1} / \mathrm{FVC}<70 \%$ & $3(4)$ & $0(0)$ & $3(8)$ & 0.116 \\
\hline $\mathrm{MMEF}_{75 / 25}$ & $85.90 \pm 24.25$ & $87.73 \pm 24.34$ & $84.03 \pm 24.32$ & 0.496 \\
\hline MMEF $_{75 / 25}<65 \%$ & $16(20)$ & $8(20)$ & $8(20)$ & 0.956 \\
\hline$D_{\text {LCO }} \%$ pred & $82.60 \pm 23.80$ & $88.13 \pm 28.11$ & $76.93 \pm 16.91$ & 0.033 \\
\hline$D_{\mathrm{LCO}}<80 \%$ pred & $44(54)$ & $17(42)$ & $27(68)$ & 0.019 \\
\hline$D_{\mathrm{Lco}} / V_{\mathrm{A}} \%$ pred & $86.10 \pm 15.72$ & $88.72 \pm 17.21$ & $83.41 \pm 13.74$ & 0.128 \\
\hline$D_{\mathrm{LCO}} / V_{\mathrm{A}}<80 \%$ pred & $31(38)$ & $14(34)$ & $17(43)$ & 0.439 \\
\hline
\end{tabular}

Data are presented as $\mathrm{n}$, mean \pm SD or $\mathrm{n}(\%)$, unless otherwise stated. BMI: body mass index; CHD: coronary heart disease; CRD: chronic respiratory disease; TLC: total lung capacity; RV: residual volume; FVC: forced vital capacity; $\mathrm{FEV}_{1}$ : forced expiratory volume in $1 \mathrm{~s} ; \mathrm{MMEF}_{75 / 25}$ : maximum mid-expiratory flow at $25-75 \%$ of FVC; $D_{\mathrm{LCO}}$ : diffusing capacity of the lung for carbon monoxide; $V_{\mathrm{A}}$ : alveolar volume.

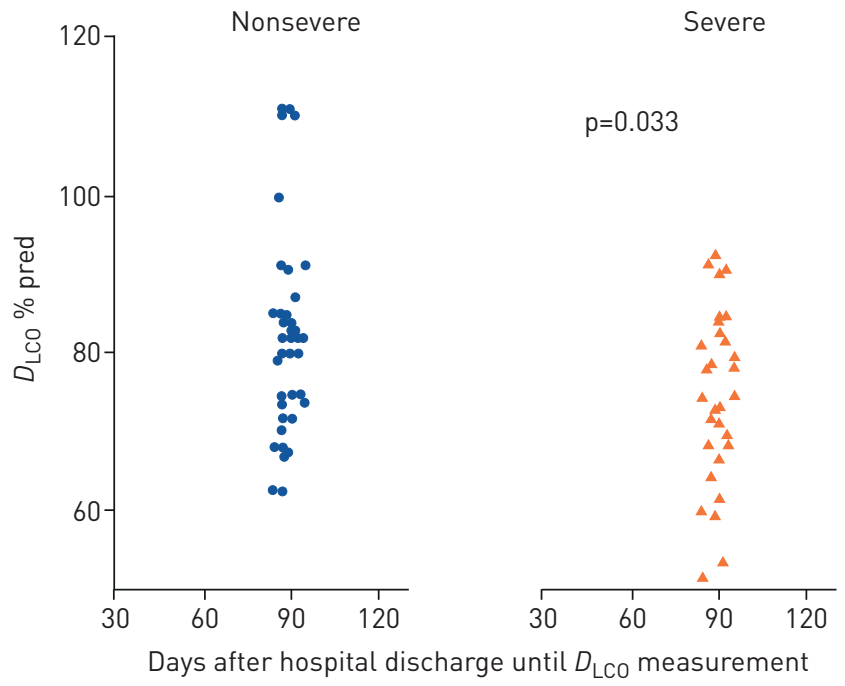

FIGURE 1 Percentage of predicted diffusing capacity of the lung for carbon monoxide $\left(D_{\text {LCOO }}\right)$ at 3 months after discharge in nonsevere and severe coronavirus disease 2019 patients. 
TABLE 2 Characteristics in coronavirus disease 2019 patients with normal and impaired diffusing capacity of the lung for carbon monoxide ( $\left.D_{\text {LCO }}\right)$ at 3-month follow-up

\begin{tabular}{|c|c|c|c|c|}
\hline & Total & Normal $D_{\text {LCo }}$ & Impaired $D_{\text {LCO }}$ & $\mathrm{p}$-value \\
\hline Patients & 81 & 37 & 44 & \\
\hline \multicolumn{5}{|l|}{$\begin{array}{l}\text { Demographics and clinical } \\
\text { characteristics }\end{array}$} \\
\hline Age years & $59(14)$ & $58 \pm 14$ & $60 \pm 14$ & 0.458 \\
\hline Male & $34(42)$ & $12(32)$ & $22(50)$ & 0.111 \\
\hline SOFA score & $1(0-2)$ & $1(0-2)$ & $1(0-3)$ & 0.217 \\
\hline $\mathrm{BMI} \mathrm{kg} \cdot \mathrm{m}^{-2}$ & $23.87 \pm 3.18$ & $23.86 \pm 3.36$ & $23.88 \pm 3.09$ & 0.977 \\
\hline Severity & & & & 0.019 \\
\hline Severe & $40(49)$ & $13(35)$ & $27(61)$ & \\
\hline Nonsevere & $41(51)$ & $24(65)$ & $17(39)$ & \\
\hline Padua score & $4(1-5)$ & $2(1-5)$ & $5(1-6)$ & 0.080 \\
\hline \multicolumn{5}{|l|}{ Comorbidities } \\
\hline Hypertension & $23(28)$ & $9(24)$ & $14(32)$ & 0.456 \\
\hline Diabetes & $7(9)$ & $5(14)$ & $2(5)$ & 0.237 \\
\hline CRD & $5(6)$ & $2(5)$ & $3(7)$ & 1.000 \\
\hline Tumour & $2(3)$ & $1(3)$ & $1(2)$ & 1.000 \\
\hline \multicolumn{5}{|l|}{$\begin{array}{l}\text { Laboratory findings on } \\
\text { admission }\end{array}$} \\
\hline WBC $\times 10^{9}$ cells $\cdot \mathrm{L}^{-1}$ & $4.98(3.80-6.28)$ & $5.54(4.46-6.94)$ & $5.39(4.35-6.94)$ & 0.894 \\
\hline Lymphocytes $\times 10^{9}$ cells $\cdot L^{-1}$ & $0.90(0.65-1.22)$ & $1(0.82-1.35)$ & $0.79(0.60-1.05)$ & 0.014 \\
\hline HCRP $\mathrm{mg} \cdot \mathrm{L}^{-1}$ & $33.31(8.71-83.79)$ & $30.08(8.33-79.84)$ & $51.90(17.49-136.20)$ & 0.157 \\
\hline NT-proBNP pg.mL ${ }^{-1}$ & $286.4(95.8-566.55)$ & $194.5(92.15-537.10)$ & $445.85(85.7-882.85)$ & 0.286 \\
\hline $\begin{array}{l}\text { Creatine kinase myocardial } \\
\text { band } U \cdot L^{-1}\end{array}$ & $10.45(7.7-13.6)$ & $9.9(7.285-12.64)$ & $11.55(8.47-15.31)$ & 0.105 \\
\hline D-dimer, $\mathrm{mg} \cdot \mathrm{L}^{-1}$ & $0.4(0.31-0.62)$ & $0.41(0.32-0.60)$ & $0.47(0.38-0.82)$ & 0.471 \\
\hline Fibrinogen $\mathrm{g} \cdot \mathrm{L}^{-1}$ & $3.91(2.96-4.69)$ & $4(3.45-5.12)$ & $4.05(2.88-4.83)$ & 0.560 \\
\hline Platelets $\times 10^{9}$ cells $\cdot \mathrm{L}^{-1}$ & $187(141-235)$ & $202(174-258)$ & $163(133-223)$ & 0.032 \\
\hline \multicolumn{5}{|l|}{ Chest CT scan on admission } \\
\hline Unilateral & $8(10)$ & $5(14)^{+}$ & $3(7)$ & 0.459 \\
\hline Bilateral & $73(90)$ & $32(87)^{+}$ & $41(93)$ & \\
\hline Unilobar & $5(6)$ & $3(8)$ & $2(5)^{+}$ & 0.656 \\
\hline Multilobar ${ }^{\#}$ & $76(94)$ & $34(92)$ & $42(96)^{+}$ & \\
\hline TSS & $9(5-13)$ & $7(2-10)$ & $12(8.25-15)$ & $<0.001$ \\
\hline MPA & $26.05 \pm 3.26$ & $25.16 \pm 3.16$ & $26.80 \pm 3.19$ & 0.023 \\
\hline AAo & $30.33 \pm 3.71$ & $29.97 \pm 3.83$ & $30.62 \pm 3.63$ & 0.433 \\
\hline MPA/AAo & $0.86 \pm 0.09$ & $0.84 \pm 0.07$ & $0.88 \pm 0.10$ & 0.053 \\
\hline \multicolumn{5}{|l|}{$\begin{array}{l}\text { Treatments during } \\
\text { hospitalisation }\end{array}$} \\
\hline Corticosteroids & $17(21)$ & 7 (19) & $10(23)$ & 0.675 \\
\hline LMWH $^{\bullet}$ & $33(41)$ & $13(35)$ & $20(46)$ & 0.346 \\
\hline HFNC & $13(16)$ & $5(14)$ & $8(18)$ & 0.569 \\
\hline Noninvasive MV & $32(40)$ & $12(32)$ & $20(46)$ & 0.232 \\
\hline ARDS & $24(30)$ & $6(16)$ & $18(41)$ & 0.015 \\
\hline \multicolumn{5}{|c|}{$\begin{array}{l}\text { Data are presented as } \mathrm{n}, \mathrm{n}(\%) \text {, median (interquartile range) or mean } \pm \text { SD, unless otherwise stated. SOFA: } \\
\text { Sequential Organ Failure Assessment; BMI: body mass index; CRD: chronic respiratory disease; WBC: white } \\
\text { blood cells; HCRP: hypersensitive C-reactive protein; NT-proBNP: N-terminal pro-brain natriuretic peptide; CT: } \\
\text { computed tomography; TSS: total severity score; MPA: main pulmonary artery; AAo: ascending aorta; LMWH: } \\
\text { low molecular weight heparin; HFNC: transnasal hyperflow oxygen therapy; MV: mechanical ventilation; ARDS: } \\
\text { acute respiratory distress syndrome. \#: }: \geqslant 2 \text { lung lobes; }{ }^{\text {I: }} \text { : used for prevention of venous thrombosis; }{ }^{+}: \text {the } \\
\text { summed percentage may exceed } 100 \% \text { due to rounding. }\end{array}$} \\
\hline
\end{tabular}

\section{Predictors for lung function decline}

Univariable logistic analysis showed that severity, TSS >10.5, MPA diameter at admission and ARDS were significantly associated with impaired $D_{\text {LCO }}$. Other variables were not associated with $D_{\text {LCo }}$ decline. Finally, we put age, MPA diameter, TSS $>10.5$ based on the ROC curve and ARDS into the multivariable logistic regression model. Details of univariable and multivariable analyses are shown in supplementary table S4 and figure 3. We found that TSS >10.5 (OR 10.4, 95\% CI 2.5-44.1; $\mathrm{p}=0.001$ ) and ARDS (OR 4.6, 95\% CI 1.4-15.5; $\mathrm{p}=0.014$ ) were significantly associated with impaired $D_{\text {LCO. }}$. 


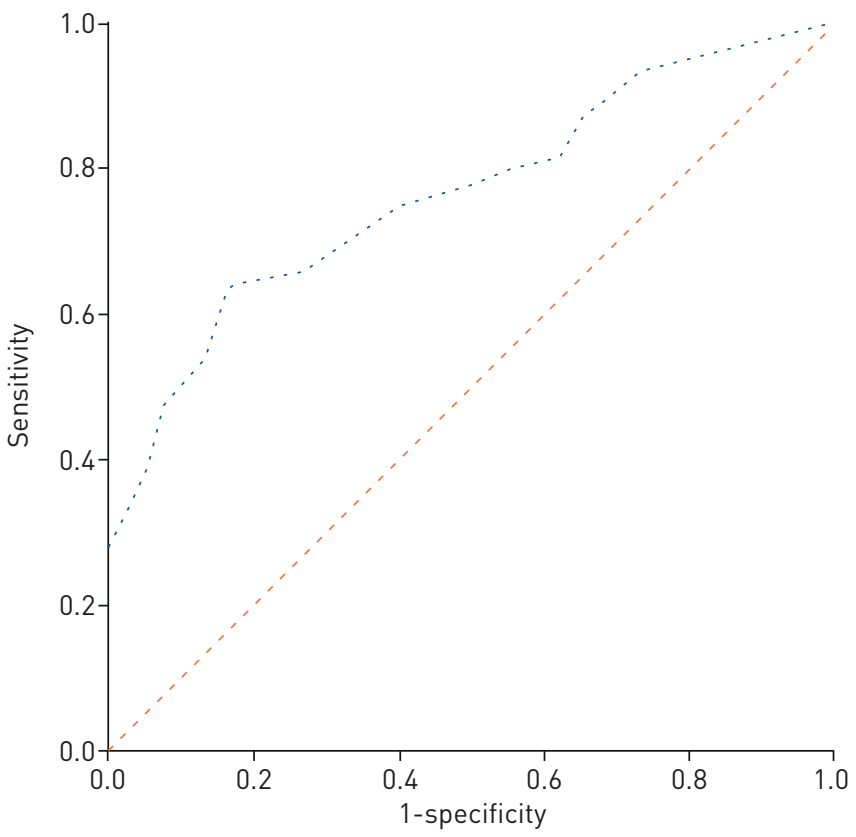

FIGURE 2 Receiver operating characteristic curve analysis of total computed tomography severity score (TSS) on admission for prediction of impaired diffusing capacity of the lung for carbon monoxide during 3-month follow-up. With the cut-off value of 10.5 for the TSS, the area under the curve was $0.765(95 \% \mathrm{Cl} 0.663-0.867$; $p<0.001$ ) with sensitivity $64 \%$ and specificity $84 \%$.

\section{Discussion}

In the present study, we focused on investigating the residual symptoms and pulmonary functions in COVID-19 patients after hospital discharge. Our study revealed that the most common residual symptoms were weakness (13\%), palpitations (10\%) and dyspnoea (9\%). Prevalences of these three symptoms were significantly higher in severe COVID-19 patients than that in nonsevere patients. In addition, COVID-19 patients presented with abnormal pulmonary function, especially impaired $D_{\text {LCO }}$ during recovery. Furthermore, patients with severe COVID-19 were found to have a higher prevalence of impaired $D_{\text {LCo. }}$. Finally, multivariable analysis in our study demonstrated that TSS $>10.5$ and ARDS were significantly associated with impaired $D_{\text {LCO }}$. Pulmonary interstitial damage may contribute to impaired $D_{\text {LCO }}$ at 3 months after discharge.

TABLE 3 Pulmonary computed tomography scan and impaired diffusing capacity of the lung for carbon monoxide $\left(D_{\mathrm{LCO}}\right)$ at 3 months

\begin{tabular}{|c|c|c|c|c|}
\hline & Total & Normal $D_{\text {LCo }}$ & Impaired $D_{\mathrm{Lco}} \#$ & $\mathrm{p}$-value \\
\hline Patients & 45 & 16 & 29 & \\
\hline Pulmonary interstitial damage & $32(71)$ & $8(50)$ & $24(83)$ & $0.037^{\star}$ \\
\hline Fibrous stripe & $23(51)$ & $7(44)$ & $16(55)$ & 0.463 \\
\hline GGO & $9(20)$ & $2(13)$ & $7(24)$ & 0.465 \\
\hline Consolidation & $5(11)$ & $1(6)$ & $4(14)$ & 0.636 \\
\hline Traction bronchiectasis & $14(31)$ & $1(6)$ & $13(45)$ & $0.008^{*}$ \\
\hline Subpleural curvilinear shadow & $22(49)$ & $2(13)$ & $20(69)$ & $<0.001^{\star}$ \\
\hline Coarse reticular pattern & $7(16)$ & $0(0)$ & $7(24)$ & $0.04^{\star}$ \\
\hline \multicolumn{5}{|l|}{ Pulmonary vascular parameters } \\
\hline MPA & $26.09 \pm 2.79$ & $25.52 \pm 2.94$ & $26.40 \pm 2.70$ & 0.327 \\
\hline AAo & $30.21 \pm 3.29$ & $29.67 \pm 3.54$ & $30.50 \pm 3.17$ & 0.442 \\
\hline MPA/AAo & $0.87 \pm 0.08$ & $0.86 \pm 0.09$ & $0.87 \pm 0.08$ & 0.855 \\
\hline
\end{tabular}


Variables

TSS > 10.5

MPA diameter

ARDS

Age years

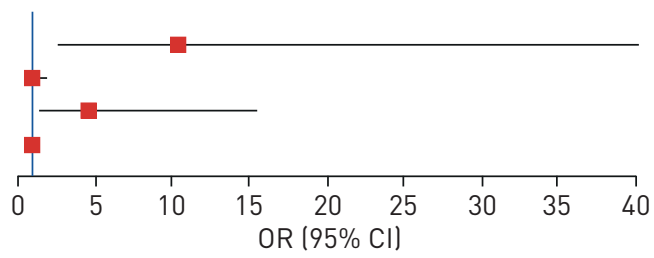

OR $(95 \% \mathrm{CI})$

$10.4(2.5-44.1)$

$1.0(0.8-1.2)$

$4.6(1.4-15.5)$

$0.99(0.95-1.03)$ p-value

0.001

0.838

0.014

0.721

FIGURE 3 Factors associated with impaired diffusing capacity of the lung for carbon monoxide $\left(D_{\mathrm{Lco}}\right)$ during 3-month follow-up in multivariable regression analysis. TSS: total severity score; MPA: main pulmonary artery; ARDS: acute respiratory distress syndrome.

During the initial epidemic of COVID-19, the most common symptoms at the onset of illness were fever, cough, fatigue and shortness of breath $[12,13]$. Some symptoms may continue following hospital discharge. It has been reported that weakness is common after acute lung injury and is associated with substantial impairments in physical function and quality of life [14]. The potential cause of these sequelae was multiple organ injury following SARS-CoV-2 infection. A prior study has shown that COVID-19 patients can have impaired physical functioning when discharged home, even after early physiotherapy [15]. For patients with SARS and MERS, the 6-min walk distance was reduced at 3 months after hospital discharge, but could be slowly improved by 12 months [16, 17]. Fatigue was reported for at least one-third of patients when followed-up for 18 months [18] and 40 months [19]. In this study, 10\% patients experienced palpitations. It was well reported that myocardial injury was common in COVID-19 patients. According to autopsy findings, viraemia in six out of 10 and five out of 12 patients demonstrated high viral RNA titres in the liver, kidney or heart [20]. In addition, myocardial injury was highly associated with fatal outcomes in this infectious disease [21, 22]. Different degrees of myocardial injury might be a reason for palpitations.

Reports by Mo et al. [3] and HuAng et al. [2] showed $D_{\text {LCO }}$ dysfunction in COVID-19 patients at discharge and 1 month from discharge, respectively. According to their studies, anomalies were noted in $D_{\text {LCO }} \%$ pred in $47.2 \%$ and $52.6 \%$ of patients, respectively. They all reported the significant difference in impaired diffusing capacity among the different groups of severity. In our study, 44 (54\%) patients had impaired diffusing capacity and there was a significant difference between nonsevere and severe COVID-19 patients at 3-month follow-up, which is in agreement with previous studies. Lung function disorder is one of the common issues with SARS and MERS patients. Pulmonary function defects were detected in half of the recovered SARS patients 3 months after hospital discharge [23]. Interstitial or pulmonary vascular abnormalities are associated with reduced $D_{\text {LCO }}$ [24], but it is unclear whether impaired $D_{\text {LCO }}$ in COVID-19 is due to pulmonary interstitial or pulmonary vascular disease, or both. In order to illuminate the reason for impaired $D_{\text {LCO }}$ in COVID-19, we analysed the TSS score on admission, pulmonary interstitial abnormalities at 3 months after discharge and markers of vasculopathy (including D-dimer, Padua score, MPA diameter, MPA/AAo ratio and use of low molecular weight heparin) and explored their associations with $D_{\text {LCO }}$ decline. As a method to score the severity of inflammation on CT images [11], TSS $>10.5$ was found to be significantly associated with impaired $D_{\text {LCO }}$, indicating that the severity of pulmonary inflammation may be the reason for impaired $D_{\text {LCO }}$. The results implied that we should follow-up the COVID-19 patients for pulmonary function, especially those individuals with high TSS upon chest CT. Additionally, we found that patients with impaired $D_{\text {LCO }}$ had a higher percentage of interstitial lesions, indicating that pulmonary interstitial damage may contribute to impaired $D_{\text {LCO }}$ at 3 months after discharge. There was no significant difference in vascular diseases between impaired and normal $D_{\text {LCO }}$ at 3-month follow-up. However, due to small sample size and lack of CT pulmonary angiography, the results could not accurately reflect the relationship between vascular abnormalities and impaired $D_{\mathrm{LCO}}$. The pathogenesis of impaired $D_{\text {LCO }}$ in COVID-19 merits further study in the future.

We analysed inherent relationships of corticosteroids treatment, inflammation on admission and ARDS occurrence with impaired $D_{\text {LCO }}$. As a result, ARDS occurrence contributed to impaired $D_{\text {LCO }}$, which was consistent with previous reports that ARDS survivors had striking decline in $D_{\text {LCO }}$, the most common abnormality in pulmonary function $[25,26]$. The exact pathologic causes of lung dysfunction in recovered COVID-19 patients remain unknown. Structural pulmonary damage caused by the ARDS and subsequent chronic changes may damage gas exchange [27]. Furthermore, neuromuscular weakness may also contribute to the impaired pulmonary function [28]. 
Although large number of patients were followed-up in our cohort, there were several limitations to our study. Firstly, pulmonary function testing was not carried out for all patients and not all patients undergoing lung function test received a chest CT scan at 3-month follow-up, mainly attributed to our limited knowledge of this novel virus and poor awareness about its impact on patients' lung function in the early epidemic era. This is the inherent limitation of this real-world study. Secondly, there was a lack of CT pulmonary angiography and other instruments to evaluate cardiovascular conditions. Furthermore, there was no direct evidence to explain the aetiology of sequelae and impaired $D_{\text {LCO }}$ in COVID-19 survivors. Although psychiatric and traumatic stress disorders were reported for patients with SARS and MERS [29, 30], a larger study with long-term follow-up needs to be carried out.

\section{Conclusion}

Weakness, palpitations and dyspnoea were the most common sequelae of COVID-19. Lung carbon monoxide diffusion dysfunction was the major damage in pulmonary function of COVID-19 survivors at 3 months after discharge. Chest CT TSS $>10.5$ and ARDS occurrence in COVID-19 were associated with

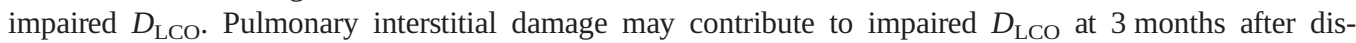
charge. This indicates that there is a necessity to adopt pulmonary rehabilitation strategy to improve outcomes in COVID-19 patients.

Acknowledgements: We thank Min Liu, Ziming Wang, Di Xu and Wei Yu (Affiliated Hospital of Jianghan University, Wuhan, China), for their data collection. We also thank Bruce L. Davidson, from the Division of Pulmonary and Critical Care Medicine, Providence Health System (Seattle, WA, USA), for his suggestions and language editing. They were not compensated for their contributions.

Author contributions: W. Qin, S. Chen, Y. Zhang, B. Hu, Z. Zhang and C. Li conceived the study. W. Qin, B. Hu, S. Chen, Z. Zhu, F. Li, X. Wang, Y. Zhang, Y. Wang, K. Zhen, J. Wang, Y. Wan and H. Li collected data. W. Qin, F. Dong, Z. Zhang, I. Elalamy, C. Li and Z. Zhai analysed and interpreted data. W. Qin, F. Dong, Y. Zhang and S. Chen drafted the manuscript. I. Elalamy, C. Li, Z. Zhai and C. Wang revised the manuscript. Z. Zhai, C. Li and C. Wang obtained funding and supervised the study.

Conflict of interest: None declared.

Support statement: This study was supported by a grant from the Chinese Academy of Engineering emergency research and cultivation project for COVID-19 (2020-KYGG-01-05), National Key Research and Development Program of China (number 2016YFC0905600; 2016YFC0901104; 2018YFC1315100), CAMS Innovation Fund for Medical Sciences (CIFMS) (number 2018-I2M-1-003) and National Natural Science Foundation of China (number 81570049; 81970058). The funding bodies are not involved in the design of the study, collection, analysis, interpretation of data or in writing the manuscript. Funding information for this article has been deposited with the Crossref Funder Registry.

\section{References}

1 Kouri A, Gupta S, Yadollahi A, et al. Addressing reduced laboratory-based pulmonary function testing during a pandemic. Chest 2020; 158: 2502-2510.

2 Huang $\mathrm{Y}$, Tan C, Wu J, et al. Impact of coronavirus disease 2019 on pulmonary function in early convalescence phase. Respir Res 2020; 21: 163.

3 Mo X, Jian W, Su Z, et al. Abnormal pulmonary function in COVID-19 patients at time of hospital discharge. Eur Respir J 2020; 55: 2001217.

4 Ahmed H, Patel K, Greenwood DC, et al. Long-term clinical outcomes in survivors of severe acute respiratory syndrome and Middle East respiratory syndrome coronavirus outbreaks after hospitalisation or ICU admission: a systematic review and meta-analysis. J Rehabil Med 2020; 52: jrm00063.

5 Beijing Respiratory Experts Panel of the Medical Staff Severe Acute Respiratory Syndrome Patients. [A follow-up study of the lung function and the chest CT changes in medical staff with severe acute respiratory syndrome in Beijing]. Zhonghua Jie He He Hu Xi Za Zhi 2005; 28: 10-12.

6 Chan KS, Zheng JP, Mok YW, et al. SARS: prognosis, outcome and sequelae. Respirology 2003; 8: Suppl. 1, S36-S40.

7 National Health Commission of the People's Republic of China. Chinese Management Guideline for COVID-19 (Trial Version 5 Revised). www.nhc.gov.cn/yzygj/s7653p/202002/d4b895337e19445f8d728fcaf1e3e13a.shtml Date last accessed: 05 February 2020. Date last updated: 08 February 2020.

8 World Health Organization. Clinical Management of Severe Acute Respiratory Infection (SARI) when COVID-19 Disease is Suspected: Interim Guidance, 13 March 2020.Geneva,World Health Organization,2020. https://apps. who.int/iris/handle/10665/331446 
9 Yu M, Liu Y, Xu D, et al. Prediction of the development of pulmonary fibrosis using serial thin-section CT and clinical features in patients discharged after treatment for COVID-19 pneumonia. Korean J Radiol 2020; 21: 746-755.

10 Li X, Zhang C, Sun X, et al. Prognostic factors of pulmonary hypertension associated with connective tissue disease: pulmonary artery size measured by chest CT. Rheumatology 2020; 59: 3221-3228.

11 Li K, Fang Y, Li W, et al. CT image visual quantitative evaluation and clinical classification of coronavirus disease (COVID-19). Eur Radiol 2020; 30: 4407-4416.

12 Huang C, Wang Y, Li X, et al. Clinical features of patients infected with 2019 novel coronavirus in Wuhan, China. Lancet 2020; 395: 497-506.

13 Chen N, Zhou M, Dong X, et al. Epidemiological and clinical characteristics of 99 cases of 2019 novel coronavirus pneumonia in Wuhan, China: a descriptive study. Lancet 2020; 395: 507-513.

14 Fan E, Dowdy DW, Colantuoni E, et al. Physical complications in acute lung injury survivors: a two-year longitudinal prospective study. Crit Care Med 2014; 42: 849-859.

15 Belli S, Balbi B, Prince I, et al. Low physical functioning and impaired performance of activities of daily life in COVID-19 patients who survived the hospitalisation. Eur Respir J 2020; 56: 2002096.

16 Hui DS, Wong KT, Ko FW, et al. The 1-year impact of severe acute respiratory syndrome on pulmonary function, exercise capacity, and quality of life in a cohort of survivors. Chest 2005; 128: 2247-2261.

17 Li TS, Gomersall CD, Joynt GM, et al. Long-term outcome of acute respiratory distress syndrome caused by severe acute respiratory syndrome (SARS): an observational study. Crit Care Resusc 2006; 8: 302-308.

18 Lee SH, Shin HS, Park HY, et al. Depression as a mediator of chronic fatigue and post-traumatic stress symptoms in Middle East respiratory syndrome survivors. Psychiatry Investig 2019; 16: 59-64.

19 Lam MH, Wing YK, Yu MW, et al. Mental morbidities and chronic fatigue in severe acute respiratory syndrome survivors: long-term follow-up. Arch Intern Med 2009; 169: 2142-2147.

20 Wichmann D, Sperhake JP, Lütgehetmann M, et al. Autopsy findings and venous thromboembolism in patients with COVID-19. Ann Intern Med 2020; 173: 268-277.

21 Shi S, Qin M, Shen B, et al. Association of cardiac injury with mortality in hospitalized patients with COVID-19 in Wuhan, China. JAMA Cardiol 2020; 5: 802-810.

22 Guo T, Fan Y, Chen M, et al. Cardiovascular implications of fatal outcomes of patients with coronavirus disease 2019 (COVID-19). JAMA Cardiol 2020; 5: 811-818.

23 Ong KC, Ng AW, Lee LS, et al. Pulmonary function and exercise capacity in survivors of severe acute respiratory syndrome. Eur Respir J 2004; 24: 436-442.

24 Ayers LN, Ginsberg ML, Fein J, et al. Diffusing capacity, specific diffusing capacity and interpretation of diffusion defects. West J Med 1975; 123: 255-264.

25 Masclans JR, Roca O, Muñoz X, et al. Quality of life, pulmonary function, and tomographic scan abnormalities after ARDS. Chest 2011; 139: 1340-1346.

26 McHugh LG, Milberg JA, Whitcomb ME, et al. Recovery of function in survivors of the acute respiratory distress syndrome. Am J Respir Crit Care Med 1994; 150: 90-94.

27 Neff TA, Stocker R, Frey HR, et al. Long-term assessment of lung function in survivors of severe ARDS. Chest 2003; 123: 845-853.

28 Ong KC, Ng AW, Lee LS, et al. 1-year pulmonary function and health status in survivors of severe acute respiratory syndrome. Chest 2005; 128: 1393-1400.

29 Mak IW, Chu CM, Pan PC, et al. Long-term psychiatric morbidities among SARS survivors. Gen Hosp Psychiatry 2009; 31: 318-326.

30 Hong X, Currier GW, Zhao X, et al. Posttraumatic stress disorder in convalescent severe acute respiratory syndrome patients: a 4-year follow-up study. Gen Hosp Psychiatry 2009; 31: 546-554. 